\title{
Clinical results of Gamma Knife surgery for cavernous sinus hemangiomas
}

\author{
Clinical article
}

\author{
*Peng Li, M.D., Haibo Ren, M.D., Shizhen Zhang, M.D., and Wei Wang, M.D. \\ Department of Neurosurgery, West China Hospital of Sichuan University, Chengdu, Sichuan Province, China
}

\begin{abstract}
Object. The purpose of this study was to gain an understanding of the efficacy and safety of Gamma Knife surgery (GKS) for the treatment of cavernous sinus hemangiomas (CSHs). The authors report on 16 patients who underwent GKS as a primary or adjuvant treatment for CSH.

Methods. Sixteen patients harboring CSHs (14 women and 2 men ranging in age from 21 to 65 years [mean 41.3 years]) underwent GKS at West China Hospital. In 4 patients the diagnosis was based on histological findings; in the other 12 patients it was based on findings on MR images. After patients were given a local anesthetic agent, the treatments were performed using a Leksell Gamma Knife model C. Gadolinium-enhanced MR images of T1-weighted, T2-weighted, and FLAIR sequences were obtained to determine the pretreatment location of the lesion. A mean peripheral dose of $13.3 \mathrm{~Gy}$ (range 11-14 Gy) was directed to the $40 \%-50 \%$ isodose line.

Results. The mean follow-up time in this study was 21.5 months (range 12-36 months). In 11 of 12 patients with symptoms, clinical improvement was reported at an average of 3.3 months (range 1-8 months) after GKS. Significant or partial tumor shrinkage was observed in 14 patients $(87.5 \%)$ at the last follow-up. No new neurological impairments were reported after GKS.

Conclusions. Magnetic resonance imaging may play an important role in the preoperative diagnosis of CSHs. Gamma Knife surgery may be a safe and effective primary or adjuvant treatment option for CSHs; however, longterm follow-up with more cases is needed to verify the benefits of this treatment. (http://thejns.org/doi/abs/10.3171/2012.7.GKS12992)
\end{abstract}

\section{KeY Words • cavernous sinus hemangioma • Gamma Knife surgery stereotactic radiosurgery}

$\mathrm{C}$ AVERNOUS sinus hemangiomas are rare benign tumors located in the region of cavernous sinus. These lesions develop as an extracerebral tumor that extends to the base of middle fossa and are considered histologically distinct from intracerebral cavernous hemangiomas. ${ }^{5}$ Because progression of CSHs is usually slow, diagnoses are incidental in some patients who are asymptomatic. Symptomatic patients may present with various neurological symptoms such as headaches, $\mathrm{CN}$ deficits, seizures, and hemorrhage..$^{10}$ Recently, the development of MR neuroimaging techniques have greatly improved the diagnosis of these types of tumors. However, management of CSHs remains difficult because these tumors have complex neurovascular structures, which may cause uncontrollable bleeding and injury to CNs when the lesions are removed..$^{10}$ Although new microsurgical

\footnotetext{
Abbreviations used in this paper: $\mathrm{CN}=$ cranial nerve; $\mathrm{CSH}=$ cavernous sinus hemangioma; GKS = Gamma Knife surgery; SRS $=$ stereotactic radiosurgery.

* Drs. Li and Ren contributed equally to this work.
}

approaches have been reported for resection of these tumors, the results are still unfavorable. , $^{45,18,25}$ In 1999, a patient harboring a residual tumor after resection underwent GKS. The tumor was controlled and the patient's clinical symptoms improved. ${ }^{7}$ Since then, more GKS cases have been reported with favorable outcomes. ${ }^{2,6,9,14,16,19,23}$ Stereotactic radiosurgery is becoming a promising option for the management of CSHs. However, reports on SRS for CSHs are sporadic and include only limited cases. To understand the efficacy and safety of this procedure, more studies are needed. Here we report on 16 patients who underwent GKS as a primary or adjuvant treatment for CSHs.

\section{Methods}

Sixteen patients with CSHs underwent GKS at West China Hospital between March 2009 and March 2011. There were 14 women and 2 men with a mean age of 41.3 years (range 21-65 years). The tumors were located on the left side in 7 cases and the right side in 9 cases. Excluding 
the 4 asymptomatic cases in which diagnosis was incidental, the most common symptom in these patients was diplopia, which occurred in 7 cases. The most frequently affected $\mathrm{CN}$ was the abducent nerve. In 4 patients, 1 of whom underwent resection and 3 of whom underwent biopsy before GKS, the diagnosis was based on histological findings. In the other 12 patients, the diagnosis was mainly based on MR images and clinical presentation. The MR images obtained in these patients showed intensely enhanced parasellar masses-iso- or hypointense on T1-weighted images and hyperintense on T2-weighted images. The characteristics of these patients are detailed in Table 1.

Treatments were performed, with the aid of a local anesthetic agent, using a Leksell Gamma Knife model C (Elekta AB). Gadolinium-enhanced MR images of T1weighted, T2-weighted, and FLAIR sequences were obtained for pretreatment localization. The mean peripheral radiation dose was 13.3 Gy (range 11-14 Gy) directed to the $40 \%-50 \%$ isodose line. The dose to the optic nerves was limited to less than $8 \mathrm{~Gy}$.

Periodic follow-up was carried out for each patient. Both in-person interviews and MR imaging were undertaken to assess changes in patients' clinical symptoms as well as tumor volume. The MR images were imported to the workstation of the Leksell Gamma Plan (version 9.0, Elekta $\mathrm{AB}$ ), where tumor volume was calculated. For a further description of the results, we classified tumor con- trol into 4 grades based on the grading scheme of Wang et al. ${ }^{22}$ Grade I was defined as an invisible tumor (disappeared); Grade II, a reduction in tumor volume that was $\geq 50 \%$ (significant shrinkage); Grade III, a reduction in tumor volume that was $\geq 20 \%$ but $<50 \%$ (partial shrinkage); and Grade IV, a reduction in tumor volume that was $<20 \%$ or an enlargement (no change). A rank sum test was used to assess the relationship between the grade of tumor control and radiation dose. The assessment of changes in clinical symptoms was based on results of the physical examination and patients' self-assessment. Obvious changes detected by physical examination or a patient assessment of $>50 \%$ improvement were classified as clinical improvement.

\section{Results}

\section{Clinical Outcomes}

The patients were followed up for an average time of 21.5 months (range 12-36 months). At the final follow-up examination, clinical improvement was reported in 11 of 12 patients with symptoms. The patient who had undergone resection previously reported unchanged ptosis and diplopia. The average interval between GKS and symptom improvement was 3.3 months (range 1-8 months). Except for a temporary headache, no newly developed neurological impairments were reported after GKS. The

TABLE 1: Characteristics of the patients enrolled in the study*

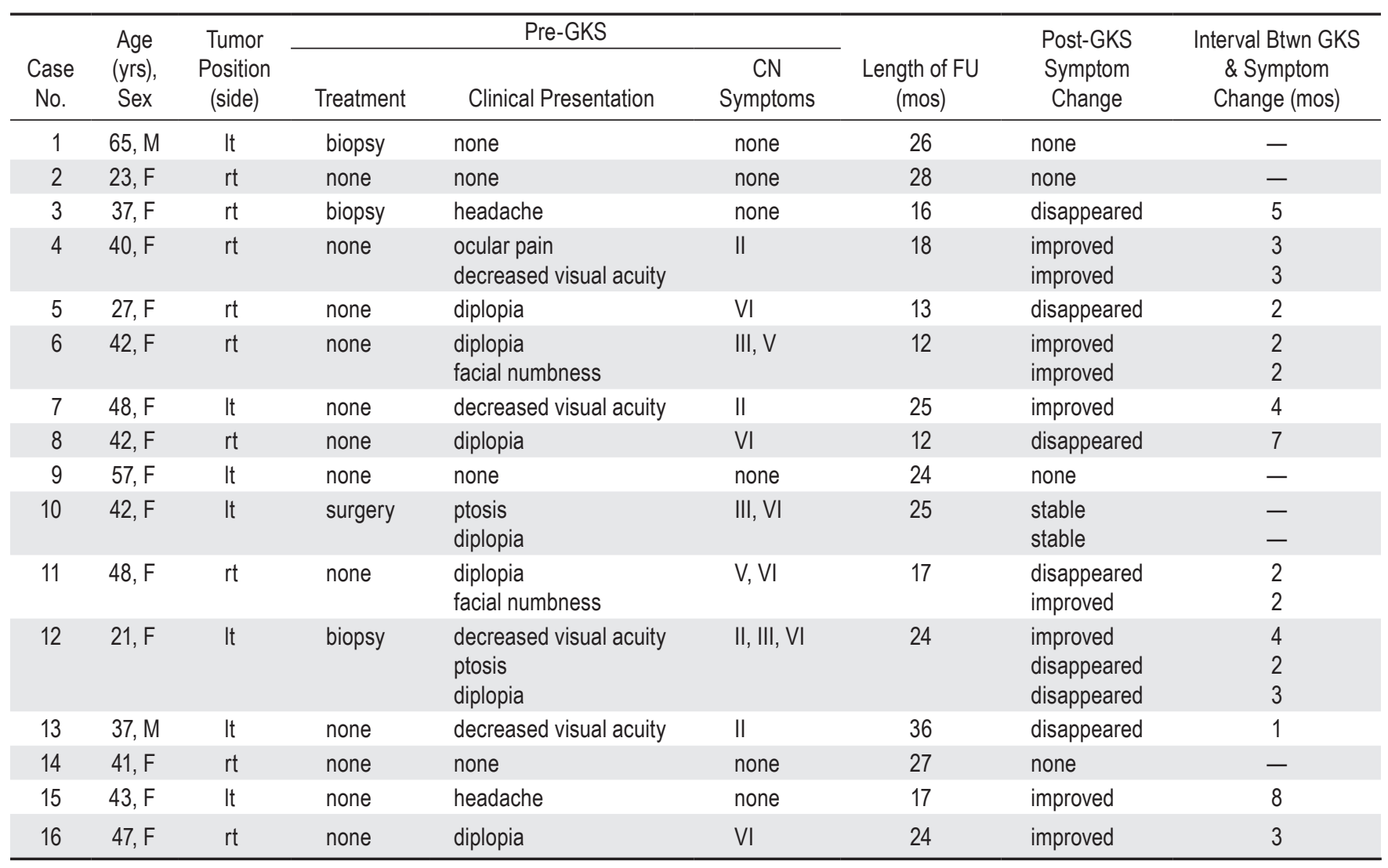

* FU = follow-up; $-=$ not applicable. 
clinical outcomes of the patients are summarized in Table 1 .

\section{Radiological Outcomes}

One year after GKS, Grade I or II tumor control was reported in 5 cases with an average reduction in tumor volume of $62.5 \%$ (range $55.0 \%-65.7 \%$ ); Grade III or IV was reported in 11 cases. Among the 9 cases still participating in follow-up 24 months after GKS, there were 1 Grade I case, 7 Grade II cases, and 1 Grade III case. Tumor shrinkage (Grades I-III) was observed in 14 patients $(87.5 \%)$ as of the last follow-up. The tumor control effect is detailed in Table 2 and Fig. 1. Figures 2 and 3 show MR images in Cases 10 and 12, respectively.

The average peripheral dose of radiation in Grade I and II cases (11 patients) was $13.6 \mathrm{~Gy}$, whereas the average dose in Grade III and IV cases (5 patients) was 12.6 Gy. The rank sum test showed a significant difference in radiation dose between Grade I-II and Grade III-IV cases $(\mathrm{p}=0.008)$.

\section{Discussion}

Cavernous sinus hemangiomas comprise up to $3 \%$ of all cavernous sinus lesions and are more frequently observed in women whose ages are between the 4th and 6th decades..$^{10}$ Similar to findings in previous reports, $87.5 \%$ $(14 / 16)$ of the patients in this cohort were female with a mean age of 42 years. The onset of CSHs is insidious. Clinical symptoms-including diplopia, ptosis, visual field defects, facial numbness, ocular pain, and headaches-are usually the results of gradual enlargement of the tumor. Cranial nerve VI was the most affected nerve both in previously reported series and in this cohort. . $^{10,1923}$ It may be because CN VI is found within the tumor, unlike CNs III, IV, or V, which usually stretch over the tu-

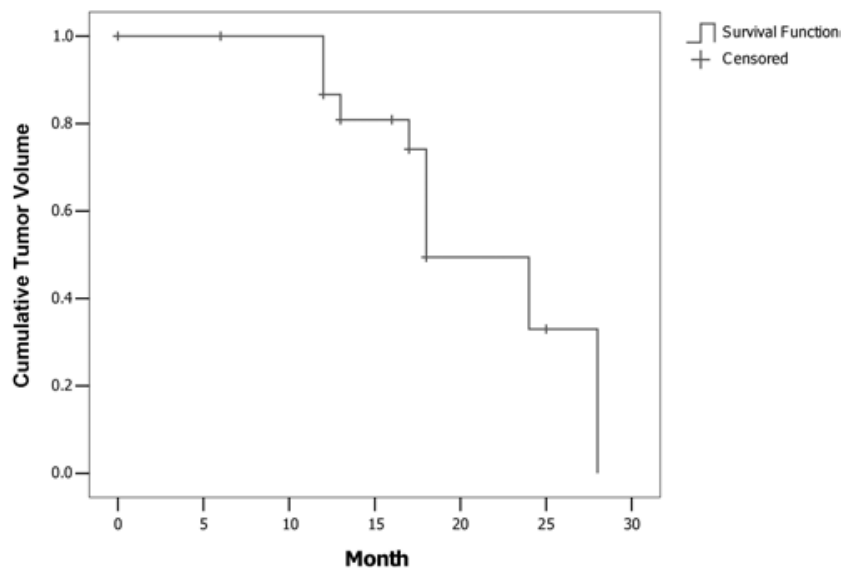

FIG. 1. Kaplan-Meier plot showing changes in tumor volume over time. A reduction in tumor volume $>50 \%$ was deemed significant.

mor surface. ${ }^{10}$ However, these symptoms are not unique for CSHs. Other lesions arising within the cavernous sinus, such as meningioma, schwannoma, and pituitary adenoma, can cause similar clinical symptoms. For this reason, a high rate of preoperative misdiagnosis was reported before the widespread use of enhanced high-resolution MR imaging. ${ }^{13}$

An asymmetrical, dumbbell-shaped lesion occupying the parasellar region and the middle cranial fossa may be discovered using CT or MR images. On CT scans, the adjacent bone structure appears normal or adsorbed, but carries no sign of the hyperplasia seen in meningiomas. ${ }^{3}$ The characteristic appearance of $\mathrm{CSH}$ on MR images reported from the 1990s greatly helped the preoperative diagnosis. ${ }^{8,10,12,24}$ The tumors may appear iso- or hypointense on T1-weighted MR images and display a significantly homogeneous or heterogeneous enhancement in

TABLE 2: Comparison of tumor volumes pre- and post-GKS

\begin{tabular}{|c|c|c|c|c|c|c|}
\hline Case No. & $\begin{array}{l}\text { Pre-GKS } \\
\text { Vol }\left(\mathrm{cm}^{3}\right)\end{array}$ & $\begin{array}{l}\text { Peripheral } \\
\text { Dose (Gy) }\end{array}$ & $\begin{array}{l}\text { Isodose } \\
\text { Line (\%) }\end{array}$ & $\begin{array}{c}\text { Post-GKS } \\
\text { Vol }\left(\mathrm{cm}^{3}\right)\end{array}$ & Vol Reduction (\%) & Grade \\
\hline 1 & 19.6 & 14 & 50 & 4.4 & 77.6 & ॥ \\
\hline 2 & 1.5 & 12 & 45 & 0.3 & 80.0 & II \\
\hline 3 & 52.7 & 11 & 50 & 40.5 & 23.1 & III \\
\hline 4 & 18.6 & 14 & 50 & 8.2 & 55.9 & $\|$ \\
\hline 5 & 17.6 & 14 & 50 & 4.4 & 75.0 & $\|$ \\
\hline 6 & 36.8 & 14 & 40 & 12.6 & 65.7 & $\|$ \\
\hline 7 & 61.5 & 13 & 50 & 33.5 & 45.5 & III \\
\hline 8 & 54.8 & 13 & 50 & 48.4 & 11.6 & IV \\
\hline 9 & 20.8 & 14 & 50 & 7.3 & 64.9 & ॥ \\
\hline 10 & 3.8 & 14 & 50 & 0 & 100 & I \\
\hline 11 & 48.6 & 13 & 45 & 26.4 & 45.6 & III \\
\hline 12 & 24.5 & 14 & 50 & 10.9 & 55.5 & $\|$ \\
\hline 13 & 14.6 & 14 & 50 & 4.5 & 69.2 & $\|$ \\
\hline 14 & 12.2 & 14 & 50 & 1.6 & 86.9 & ॥ \\
\hline 15 & 50.4 & 13 & 50 & 40.6 & 19.4 & IV \\
\hline 16 & 48.4 & 12 & 50 & 11.2 & 76.9 & II \\
\hline
\end{tabular}



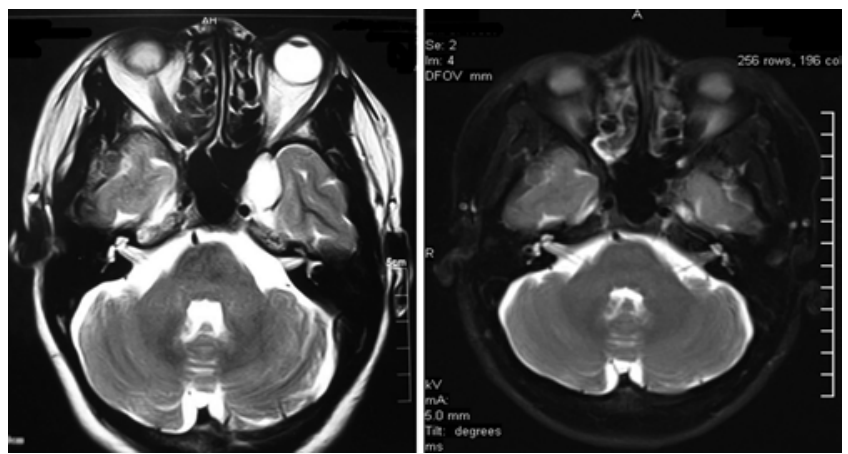

FIG. 2. Case 10. Magnetic resonance images obtained before GKS (left) and 21 months after treatment (right).

response to a gadolinium-DTPA injection. Usually, no edema reaction around the tumor can be observed. Welldefined tumor hyperintensivity on T2-weighted MR images and no "dural tail" signs on enhanced MR images can help distinguish CSHs from meningiomas. However, there are still some difficulties in distinguishing a diagnosis of CSH from those of other tumors of the cavernous sinus. The pulse sequence of FLAIR is an inversionrecovery technique that can nullify fluids on the image. The high CSF signal in T2-weighted and proton densityweighted images can be suppressed using the FLAIR sequence so that the pathological area adjacent to the CSF signal is seen more clearly. This technique has been widely used for the diagnosis for multiple sclerosis. ${ }^{1}$ However, only limited information about imaging findings of CSHs using FLAIR is available. ${ }^{20}$ Unlike other tumors arising from the cavernous sinus, all of the CSHs in our study exhibited hyperintensity on FLAIR images. The results indicate that the FLAIR sequence could be a useful tool for preoperative diagnosis of CSHs. The characteristics of MR images of CSHs are detailed in Table 3.

Since CSHs are benign tumors, cure is possible if complete resection is achieved; however, debate still remains as to what is the optimum treatment strategy. Re-
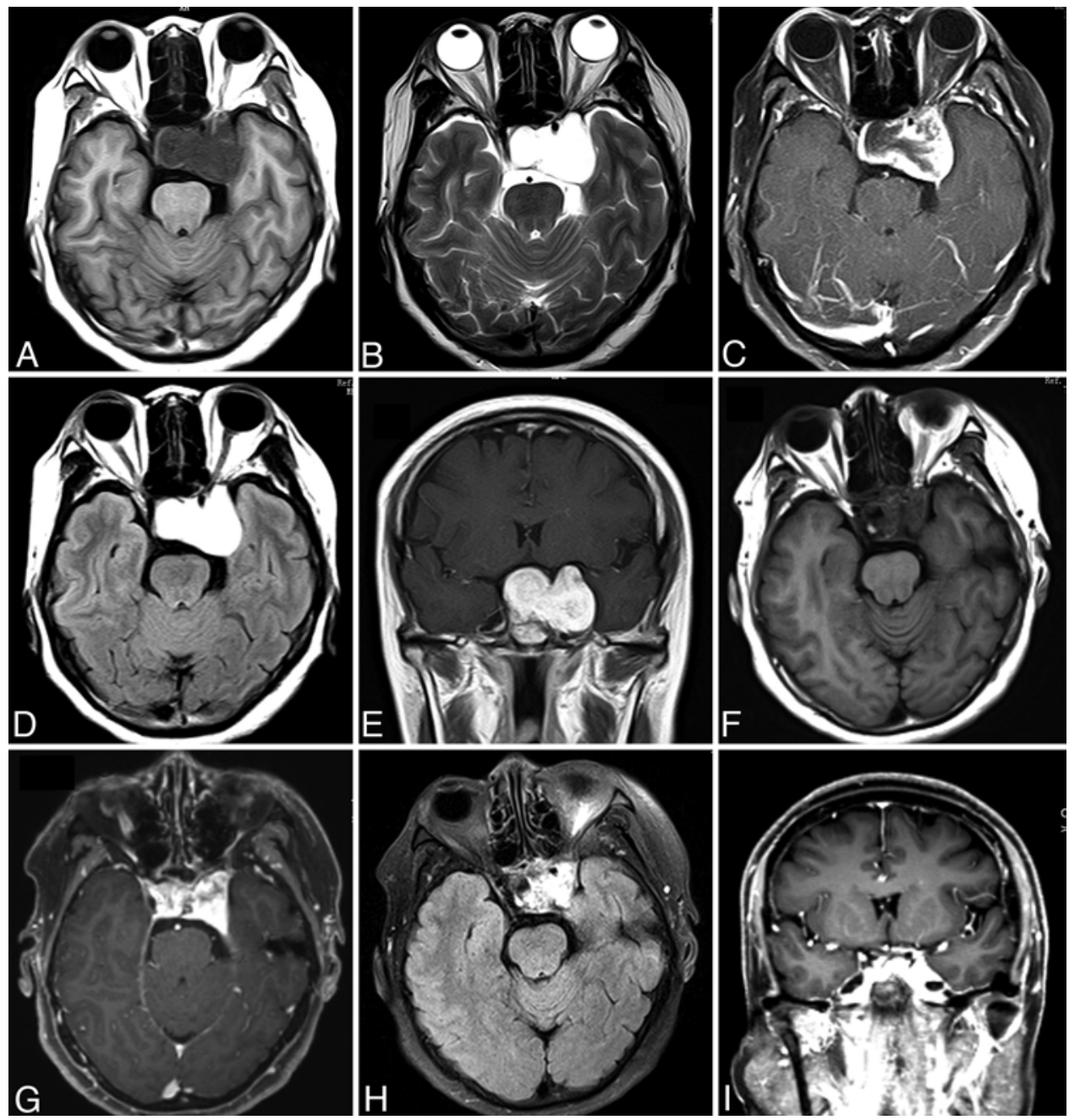

FIG. 3. Case 12. Magnetic resonance images obtained before GKS: axial T1-weighted (A), T2-weighted (B), Gd-enhanced T1weighted (C), and FLAIR (D) images, as well as a coronal enhanced T1-weighted (E) image. Additional MR images obtained 24 months after GKS: axial T1-weighted (F), Gd-enhanced T1-weighted (G), and FLAIR (H) images, as well as a coronal enhanced T1-weighted image (I). 
TABLE 3: Characteristics of CSHs on MR images*

\begin{tabular}{|c|c|c|c|c|c|}
\hline Authors \& Year & T1-Weighted & T2-Weighted & $\begin{array}{l}\text { Gd-Enhanced } \\
\text { T1-Weighted }\end{array}$ & FLAIR & Diffusion-Weighted \\
\hline Iwai et al., 1999 & $\rightarrow$ & $\uparrow$ & $\uparrow$ & - & - \\
\hline Thompson et al., 2000 & $\downarrow$ & $\uparrow$ & $\uparrow$ & - & - \\
\hline Sohn et al., 2003 & $\downarrow$ & $\uparrow$ & $\uparrow$ & - & - \\
\hline Zhou et al., 2003 & $\downarrow / \rightarrow$ & $\uparrow$ & $\uparrow$ & - & - \\
\hline Mendonça et al., 2004 & $\rightarrow$ & $\uparrow$ & $\uparrow$ & - & $\rightarrow$ \\
\hline Yao et al., 2006 & $\downarrow / \uparrow \uparrow$ & $\uparrow$ & $\uparrow$ & - & - \\
\hline Ivanov et al., 2008 & $\rightarrow$ & $\uparrow$ & $\uparrow$ & - & - \\
\hline Jinhu et al., 2008 & $\rightarrow$ & $\uparrow$ & $\uparrow / \rightarrow \ddagger$ & - & - \\
\hline Yamamoto et al., 2010 & - & - & $\uparrow$ & - & - \\
\hline present series & $\downarrow / \rightarrow$ & $\uparrow$ & $\uparrow$ & $\uparrow$ & - \\
\hline
\end{tabular}

section is recommended as a primary treatment option by some centers..$^{15,18,25}$ Nevertheless, total resection of these tumors is a great challenge because of excessive intraoperative hemorrhage and the potential for injury to $\mathrm{CNs}$ due to the complicated neurovascular structures of the cavernous sinus. Although Zhou et al. ${ }^{25}$ reported that total tumor removal was obtained in 12 of 13 patients by using the epidural approach, in most previous studies the total resection rate was only $30 \%-44 \%$ and there was a high rate of complications. ${ }^{4,15,18,25}$ In our study, severe blood loss was encountered in the 4 patients who had undergone open biopsy or partial resection. Because of the high risk of open surgery, noninvasive techniques should be considered for these patients. Since the first successfully treated case was reported by Iwai et al., 7 more reports on GKS for the treatment of CSHs have been published indicating a good response and low complication rate. ${ }^{2,6,9,14,16,19,23} \mathrm{~A}$ recently published systematic review and meta-analysis, which included 59 cases with a mean follow-up period of 49.2 months (range 6-156 months), added to the eidence that GKS can be a suitable option for the treatment of CSHs. Among the patients included in that study, tumor shrinkage was observed in 55 patients (93.2\%). Thirtyfive of the 46 symptomatic patients reported clinical improvement. Additional $\mathrm{CN}$ impairments were reported by only 1 patient..$^{22}$ Nevertheless, in most previous reports, GKS was used as adjuvant treatment after open surgery. The effects of GKS as a primary treatment have only been reported sporadically and include limited cases. The present report, to the best of our knowledge, is one of the largest series with the largest average tumor volume of $30.4 \mathrm{~cm}^{3}$ (range $1.5-61.5 \mathrm{~cm}^{3}$ ) at a single institution. In most cases in this study there were large tumors and the patients underwent GKS as a primary treatment, resulting in a good response. A review of previous reports is summarized in Table 4.

Obtaining periodic MR images is necessary to assess tumor control and provide quantitative information on tumor volume. Using the image registration function of the Leksell Gamma Plan software, tumor shrinkage was observed in 13 cases (81.2\%). Tumor shrinkage was most significant during the first 2 years after GKS. Similar results were reported by Yamamoto et al..$^{23}$ and Ivanov and colleagues. ${ }^{6}$ Rapid tumor shrinkage in our study and in previous reports provides convincing evidence that CSHs are radiosensitive. . $7,7,14,19,23$ Further studies concerning the optimum radiation dose and treatment strategy are still needed, however, especially for patients harboring large tumors. Based on our review of the literature and events in our study, a peripheral dose of 8-19 Gy was planned for CSHs, accounting for the volume of the tumors and distances from the optic pathways and adjacent brain tissues. Yamamoto et al. ${ }^{23}$ suggested that the threshold level of a peripheral dose for tumor control is 10-12 Gy and that a dose of 14-15 Gy is sufficient to control the growth of CSHs. The statistical analysis in our study indicates that a higher peripheral dose might result in better tumor control. However, the higher peripheral dose was usually delivered to patients with a smaller tumor volume to avoid radiation-related toxicity. Although many cases of large tumors in this study exhibited tumor shrinkage (Grades I-III) without inducing obvious neurological impairments, hypofractionated SRS may still be an option for these cases to increase the dose of radiation without increasing the toxicity. ${ }^{21}$

The rate of short-term and perpetual complications of GKS for CSHs is relatively low. Only 1 of 59 patients had additional trigeminal nerve disturbance in the systematic review by Wang et al. ${ }^{22}$ None of our patients developed new GKS-related neurological impairments, except for the 1 case of temporary headache, which may have been related to fixation of the head-stabilization frame. The optic chiasm and nerve are more susceptible to injury from radiotherapy than other CNs. The maximum radiation dose of $10-12$ Gy to the optic pathways is expected to lead to radiation-related optic nerve injuries in less than $2 \%$ of the patients. ${ }^{11}$ Thus a maximum radiation dose of $10 \mathrm{~Gy}$ to the optic pathways would be safe 
TABLE 4: Summary of previous reports*

\begin{tabular}{|c|c|c|c|c|c|c|c|c|c|c|c|c|}
\hline \multirow[b]{2}{*}{ Authors \& Year } & \multirow{2}{*}{$\begin{array}{l}\text { No. of } \\
\text { Cases }\end{array}$} & \multicolumn{2}{|c|}{ Previous Surgery } & \multicolumn{2}{|c|}{ Tumor Vol $\left(\mathrm{cm}^{3}\right)$} & \multirow{2}{*}{$\begin{array}{l}\text { Peripheral } \\
\text { Dose (Gy) }\end{array}$} & \multirow{2}{*}{$\begin{array}{l}\text { Length of } \\
\text { FU (mos) }\end{array}$} & \multicolumn{3}{|c|}{ Symptom Change Post-GKS } & \multicolumn{2}{|c|}{ Tumor Shrinkageł } \\
\hline & & Yes & No & Mean & Range & & & Stable & Improved & None† & Yes & No \\
\hline Iwai et al., 1999 & 1 & 1 & - & 5.3 & - & 12 & 20 & 1 & - & - & 1 & - \\
\hline Nakamura et al., 2002 & 3 & 1 & 2 & 6.5 & $3.3-9.5$ & $12-14$ & $24-60$ & 1 & 1 & 1 & 2 & 1 \\
\hline Peker et al., 2004 & 5 & 4 & 1 & 5.0 & $3.8-6.2$ & $14-16$ & $6-52$ & 2 & 2 & 1 & 5 & - \\
\hline Chou et al., 2010 & 7 & 3 & 4 & 9.9 & $2.9-23.1$ & 12.5 & $6-156$ & 1 & 5 & 1 & 7 & - \\
\hline Yamamoto et al., $2010 \S$ & 30 & 17 & 13 & 11.5 & $1.5-51.4$ & $8-17$ & $12-138$ & 7 & 15 & 8 & 29 & 1 \\
\hline present series & 16 & 4 & 12 & 30.4 & $1.5-61.5$ & $11-14$ & $12-36$ & 1 & 11 & 4 & 14 & 2 \\
\hline
\end{tabular}

* $-=$ not applicable.

$\dagger$ None $=$ no clinical symptoms pre- and post-GKS.

¥ More than $20 \%$ reduction in tumor volume was considered tumor shrinkage.

$\S$ This report included results from 7 institutions in Japan.

for treatment. Accounting for possible system errors in the Gamma Knife unit and deviation of MR images, we limited the dose to the optic nerves to less than $8 \mathrm{~Gy}$.

Given the high risk of excessive blood loss from open surgery or biopsy, no histological evidence was available for 12 patients in our study. Diagnosis was mainly dependent on typical MR imaging and CT presentation. Although CSHs have imaging characteristics that are different from other tumors, misdiagnosed cases might have been included. This is the limitation of our study.

\section{Conclusions}

Cavernous sinus hemangiomas can present a characteristic appearance on T1- and T2-weighted MR images and FLAIR sequences. Although histological evidence remains the "gold standard" for the diagnosis of CSHs, MR imaging may play an important role in the preoperative diagnosis of these lesions. Our study showed that most cases of CSHs, even those in which the tumors were large, respond well to GKS. The results of this study add evidence that GKS is a useful and safe therapeutic method for CSHs, both as a primary or adjuvant treatment. Further studies with long-term follow-up and larger numbers of cases are necessary to optimize treatment conditions and verify the benefit of this treatment.

\section{Disclosure}

The authors report no conflict of interest concerning the materials or methods used in this study or the findings specified in this paper.

Author contributions to the study and manuscript preparation include the following. Conception and design: Wang, Li. Acquisition of data: Ren, Zhang. Analysis and interpretation of data: Li, Ren. Drafting the article: Li, Ren. Critically revising the article: all authors. Reviewed submitted version of manuscript: all authors. Approved the final version of the manuscript on behalf of all authors: Wang. Statistical analysis: Ren, Zhang. Study supervision: Wang.

\section{References}

1. Bakshi R, Ariyaratana S, Benedict RH, Jacobs L: Fluid-attenuated inversion recovery magnetic resonance imaging detects cortical and juxtacortical multiple sclerosis lesions. Arch Neurol 58:742-748, 2001

2. Chou CW, Wu HM, Huang CI, Chung WY, Guo WY, Shih $\mathrm{YH}$, et al: Gamma knife surgery for cavernous hemangiomas in the cavernous sinus. Neurosurgery 67:611-616, 2010

3. Eisenberg MB: Cavernous hemangiomas of the cavernous sinus, in Eisenberg MB, Al-Mefty O (eds): The Cavernous Sinus. Philadephia: Lippincott Williams \& Wilkins, 2000, pp 315-319

4. Fraser JF, Mass AY, Brown S, Anand VK, Schwartz TH: Transnasal endoscopic resection of a cavernous sinus hemangioma: technical note and review of the literature. Skull Base 18:309-315, 2008

5. Gonzalez LF, Lekovic GP, Eschbacher J, Coons S, Porter RW, Spetzler RF: Are cavernous sinus hemangiomas and cavernous malformations different entities? Neurosurg Focus 21(1): e6, 2006

6. Ivanov P, Chernov M, Hayashi M, Nakaya K, Izawa M, Murata $\mathrm{N}$, et al: Low-dose gamma knife radiosurgery for cavernous sinus hemangioma: report of 3 cases and literature review. Minim Invasive Neurosurg 51:140-146, 2008

7. Iwai Y, Yamanaka K, Nakajima H, Yasui T: Stereotactic radiosurgery for cavernous sinus cavernous hemangioma-case report. Neurol Med Chir (Tokyo) 39:288-290, 1999

8. Jinhu Y, Jianping D, Xin L, Yuanli Z: Dynamic enhancement features of cavernous sinus cavernous hemangiomas on conventional contrast-enhanced MR imaging. AJNR Am J Neuroradiol 29:577-581, 2008

9. Khan AA, Niranjan A, Kano H, Kondziolka D, Flickinger JC, Lunsford LD: Stereotactic radiosurgery for cavernous sinus or orbital hemangiomas. Neurosurgery 65:914-918, 2009

10. Linskey ME, Sekhar LN: Cavernous sinus hemangiomas: a series, a review, and an hypothesis. Neurosurgery 30:101-108, 1992

11. Mayo C, Martel MK, Marks LB, Flickinger J, Nam J, Kirkpatrick J: Radiation dose-volume effects of optic nerves and chiasm. Int J Radiat Oncol Biol Phys 76 (3 Suppl):S28-S35, 2010

12. Mendonça JL, Viana SL, Matsumine M, Silva RF, Viana MA, Freitas FM: Cavernous angioma of the cavernous sinus: imaging findings. Arq Neuropsiquiatr 62:1004-1007, 2004 


\section{Gamma Knife surgery for cavernous sinus hemangiomas}

13. Meyer FB, Lombardi D, Scheithauer B, Nichols DA: Extraaxial cavernous hemangiomas involving the dural sinuses. J Neurosurg 73:187-192, 1990

14. Nakamura N, Shin M, Tago M, Terahara A, Kurita H, Nakagawa $\mathrm{K}$, et al: Gamma knife radiosurgery for cavernous hemangiomas in the cavernous sinus. Report of three cases. $\mathbf{J}$ Neurosurg 97 (5 Suppl):477-480, 2002

15. Ohata K, El-Naggar A, Takami T, Morino M, El-Adawy Y, ElSheik K, et al: Efficacy of induced hypotension in the surgical treatment of large cavernous sinus cavernomas. J Neurosurg 90:702-708, 1999

16. Peker S, Kiliç T, Sengöz M, Pamir MN: Radiosurgical treatment of cavernous sinus cavernous haemangiomas. Acta Neurochir (Wien) 146:337-341, 2004

17. Sohn CH, Kim SP, Kim IM, Lee JH, Lee HK: Characteristic MR imaging findings of cavernous hemangiomas in the cavernous sinus. AJNR Am J Neuroradiol 24:1148-1151, 2003

18. Suri A, Ahmad FU, Mahapatra AK: Extradural transcavernous approach to cavernous sinus hemangiomas. Neurosurgery 60:483-489, 2007

19. Thompson TP, Lunsford LD, Flickinger JC: Radiosurgery for hemangiomas of the cavernous sinus and orbit: technical case report. Neurosurgery 47:778-783, 2000

20. Toyoda K, Oba H: [Diagnostic imaging of hemangiomas in the brain.] Brain Nerve 63:5-15, 2011 (Jpn)

21. Wang X, Liu X, Mei G, Dai J, Pan L, Wang E: Phase II study to assess the efficacy of hypofractionated stereotactic radiotherapy in patients with large cavernous sinus hemangiomas. Int J Radiat Oncol Biol Phys 83:e223-e230, 2012
22. Wang X, Mei G, Liu X, Dai J, Pan L, Wang E: The role of stereotactic radiosurgery in cavernous sinus hemangiomas: a systematic review and meta-analysis. J Neurooncol 107:239_ 245, 2012

23. Yamamoto M, Kida Y, Fukuoka S, Iwai Y, Jokura H, Akabane A, et al: Gamma Knife radiosurgery for hemangiomas of the cavernous sinus: a seven-institute study in Japan. Clinical article. J Neurosurg 112:772-779, 2010

24. Yao Z, Feng X, Chen X, Zee C: Magnetic resonance imaging characteristics with pathological correlation of cavernous malformation in cavernous sinus. J Comput Assist Tomogr 30:975-979, 2006

25. Zhou LF, Mao Y, Chen L: Diagnosis and surgical treatment of cavernous sinus hemangiomas: an experience of 20 cases. Surg Neurol 60:31-37, 2003

Manuscript submitted May 15, 2012.

Accepted July 18, 2012.

Portions of this work were presented in abstract form at the 16th Annual Leksell Gamma Knife Society Meeting, Sydney, Australia, March 25, 2012.

Please include this information when citing this paper: DOI: 10.3171/2012.7.GKS12992.

Address correspondence to: Wei Wang, M.D., Department of Neurosurgery, West China Hospital of Sichuan University, 37 Guoxue Alley, Chengdu, Sichuan Province, China 610041. email: wcnslp@163.com. 